\title{
PERCEPÇÕES E DIFICULDADES DE FUMANTES NA CESSAÇÃO DO
}

\author{
HÁBITO DE FUMAR
}

Lisandra Iaponira Henrique MAIA ${ }^{1}$

Ellany Gurgel Cosme do NASCIMENTO²

\begin{abstract}
${ }^{1}$ Graduanda em enfermagem pela Universidade do Estado do Rio Grande do Norte, lisandra.iaponira@ hotmail.com.
${ }^{2}$ Enfermeira, doutoranda em Ciências da Saúde, UFRN, docente do departamento de enfermagem na Universidade do Estado do Rio Grande do Norte, UERN. Pesquisadora do grupo de pesquisa Conhecimento, Enfermagem e Saúde das Populações (Grupesces). Email- ellanygurgel @ hotmail.com. Participou da concepção, análise, interpretação do dados e revisão. Endereço: Universidade do Estado do Rio Grande do Norte-UERN, Campus Avançado Prof ${ }^{a} \mathrm{M}^{\mathrm{a}}$. Elisa de Albuquerque Maia-CAMEAM, Pau dos Ferros, RN, no endereço BR 405, KM 153, Arizona 59900-000 - Pau dos Ferros - RN. Telefone: (84) 9648-5999 (Autor para correspondência).
\end{abstract}

\section{Recebido em: 09/03/2015 - Aprovado em: 11/08/2015 - Disponibilizado em: 30/10/2015}

\begin{abstract}
RESUMO:
$\mathrm{O}$ ato de fumar envolve hábitos, comportamentos, percepções, sensações, prazeres e sentimentos que juntos constroem todo um significado atrás do simples ato de tragar um cigarro, implicando diretamente na dificuldade de muitos de largar o fumo. O objetivo desse artigo é identificar o que a literatura oferece nos últimos cinco anos sobre fumantes e dificuldades encontradas ao decidirem parar de fumar, sintetizando as principais percepções das pessoas em relação a esse comportamento a fim de fundamentar o cuidado a esta clientela. Utilizamos a revisão sistemática da literatura, na qual delimitamos as seguintes etapas; elaboração da pergunta norteadora, estabelecimento de palavras- chave e de critérios para inclusão/exclusão de artigos. Prevalecem nas publicações, as principais dificuldades encontradas pelos fumantes ao tentarem parar de fumar e principais motivos que levam as pessoas a decidirem parar de fumar. Os resultados foram organizados na forma de tabela para facilitar a observação. O estudo nos possibilitou identificar o quão difícil é trabalhar com a temática, visto que mesmo que sejam inúmeros os estudos relacionados ao tabagismo, poucos trabalham as percepções dos indivíduos em seu enfrentamento ao vicio.
\end{abstract}

Palavras-chave: percepções, fumantes, tabagismo.

\section{PERCEPTIONS AND DIFFICULTIES OF SMOKERS IN THE CESSATION}

\section{OF SMOKING}

\begin{abstract}
:
The act involves smoking habits, behaviors, perceptions, sensations, pleasures and feelings that together build a whole meaning behind the simple act of inhaling a cigarette, which directly involves difficulty for many to quit smoking. The aim of this paper is to identify what literature offers the last five years on smoking and difficulties in deciding to quit smoking, summarizing the main perceptions of people regarding this behavior in order to substantiate care to this clientele. We use a systematic literature review, in which delimit the following steps; drafting the guiding question, establishing keywords and criteria for inclusion / exclusion of articles. Prevail in the publications, the main difficulties encountered by smokers trying to quit smoking and main reasons why people decide to quit smoking. The results were organized in tabular form to facilitate observation. The study allowed us to identify how difficult it is to work with the theme, since even if they are numerous studies related to smoking, few work the perceptions of individuals in coping with the addiction.
\end{abstract}

Keywords : perceptions, smokers, smoking . 


\section{INTRODUÇÃO}

O tabagismo é atualmente reconhecido como uma dependência química, sendo classificado internacionalmente pela Organização Mundial da Saúde no grupo dos transtornos mentais e de comportamento (CID 10) decorrentes do uso de substâncias psicoativas. (OLIVEIRA, 2008).

Segundo Goulart, 2010, o desejo de parar de fumar é maior entre aqueles que reconhecem ser esse um hábito prejudicial à saúde. Isso deve ser levado em conta na adoção de estratégias que visam à redução do tabagismo. Porém mesmo que muitos saibam os efeitos negativos que o cigarro pode acarretar ao nosso organismo, o ato de fumar envolve hábitos, comportamentos, percepções, sensações, prazeres e sentimentos que juntos constroem todo um significado atrás do simples ato de tragar um cigarro, que implica diretamente na dificuldade de muitos de largar o fumo.

Considerando os aspectos mencionados, o objetivo desse artigo é identificar o que a literatura oferece nos últimos cinco anos sobre fumantes e dificuldades encontradas ao decidirem parar de fumar, sintetizando as principais percepções das pessoas em relação a esse comportamento a fim de fundamentar o cuidado a esta clientela.

\section{METODOLOGIA}

Para o alcance do objetivo proposto selecionamos como método de pesquisa, a revisão sistemática da literatura, delimitando as seguintes etapas: identificação do problema; elaboração da pergunta norteadora, estabelecimento de palavras- chave e de critérios para inclusão/exclusão de artigos. Em seguida realizamos a seleção dos artigos, e a definição das informações a serem extraídas dos trabalhos revisados, a discussão e interpretação dos resultados e, por fim, a síntese dos conhecimentos objetivos, e principais conclusões. A pesquisa foi realizada entre dezembro de 2013 a janeiro de 2014.

A busca dos artigos ocorreu na base de dados PubMed- Recurso livre do Medline desenvolvido pelo Centro Nacional de Informação sobre Biotecnologia - National Center for Biotechnology Information (NCBI) - para os Estados Unidos da América (EUA) Biblioteca Nacional de Medicina - National Library of Medicine (NLM) - que possibilita a pesquisa bibliográfica em mais de 22 milhões de artigos médicos, incluindo artigos internacionais (PUBMED, 2014) e Scientific Electronic Library Online (SCIELO)biblioteca eletrônica que abrange uma coleção selecionada de periódicos científicos brasileiros (SCIELO, 2014).

Utilizou-se como palavra- chave: smoking cessation. Chegou-se a um total de 
438.117 artigos, logo em seguida foram utilizados os critérios de inclusão e exclusão que foram: texto completo grátis disponível, dos últimos cinco anos (2008 a 2013), pesquisas com seres humanos, e que a publicação deveria conter em sua discussão a temática em estudo.

\section{RESULTADOS}

Entre as discussões elencadas, encontram-se temas voltados às percepções dos fumantes em seu desafio, desde a decisão de querer ou não parar de fumar, suas crenças sobre o fumo, principais dificuldades e formas de apoio encontradas ao decidir parar de fumar. As percepções foram organizadas na forma de tabela para facilitar a observação, a tabela 1 descreve os principais motivos que levam as pessoas a decidirem parar de fumar, à tabela 2 às principais dificuldades encontradas pelos fumantes ao tentarem parar de fumar.

Tabela 1 - Principais motivos que levam as pessoas a decidirem parar de fumar

Comportamentos preconceituosos por parte de não fumantes

Conselhos de familiares sobre a procura do serviço

Conselhos de Profissionais de saúde para o abandono do vicio

Participação ativa do familiar no tratamento

Preocupação com a saúde atual e futura

Doença relacionada com o tabaco

Preocupações financeiras

Gravidez / começar uma família

Impacto que o cigarro estaria acarretando em crianças e na saúde da família
Tabela 2 - Principais dificuldades encontradas pelos fumantes ao tentarem parar de fumar

Ausência de apoio familiar

Desestruturação familiar

Ver outras pessoas fumando

Problemas familiares e falta de apoio.

Desconhecimento quanto às formas de tratamento

Muitos fumantes não sentem os efeitos prejudiciais que o cigarro causa a saúde a curto ou médio prazo.

Ignorar ou desconhecer os resultados que o cigarro poderá ocasionar

Sentimentos como o prazer em fumar, tédio, força do hábito, estresse.

Tentação ambiental e fatores sociais

Pressão emocional, vício e comportamento habitual

\section{DISCUSSÃO}

Por que as pessoas fumam? Provavelmente a resposta para essa questão nem sempre é tão simples como muitos pensam. Para muitos é o simples fato da dependência a nicotina. Hoje estudos demonstram que a causa do tabagismo vai muito mais além da dependência dessa substância, sendo constituída de uma complexa relação entre estímulos ambientais, hábitos pessoais, condicionamentos psicossociais. (CARVALHO et al, 2010).

Muitas pessoas têm facilidade para largar o vicio, enquanto outros lutam com bastante dificuldade para largar o ciclo vicioso. As proibições de fumar em lugares públicos estão em vigor, mas isso não é suficiente para um tratamento de tabaco completo bem como única forma de prevenção. Sendo necessário que sejam inclusos programas de controle e apoio á cessação. (T. GOODFELLOW, 2009). 
Entre os principais motivos que levaram os participantes a decisão de parar de fumar e/ou procurar ajuda profissional com vistas à mudança do comportamento de fumar, encontrou-se a categoria prejuízo à própria saúde e/ou prevenção de doenças relacionadas ao tabaco. (RUSSO et al., 2010, MARTINS et al., 2011, BARRETO et al., 2012).

Entre as principais razões para a ainda elevada prevalência de fumantes decorrem do fato de que a maioria das doenças provocadas pelo tabaco são de natureza crônica, sendo que muitos fumantes tem planos para desfrutar bastante do cigarro atualmente e planejam parar de fumar no futuro. Preferem acreditar que os resultados do tabagismo só afetam os mais velhos, que já tiveram um longo tempo de uso. Somando-se ainda a propaganda veiculada pela indústria do tabaco, ao baixo preço, e pelo próprio fato de ser difícil deixar de fumar, pois a maioria dos fumantes é dependente da nicotina, além de aspectos sociais, econômicos e culturais associados. Sugere-se assim que os fumantes devem ser alvo com maior ênfase dos benefícios associados à prevenção de doenças relacionadas ao tabaco, bem como a qualidade de vida e benefícios de parar de fumar, mesmo antes do aparecimento de qualquer doença relacionada ao tabagismo. (SANTOS, 2009, AZEVEDO et al, 2011, UPPAL et al, 2013, CORNER et al, 2013)
Pacientes com alto risco com comorbidades e em processo de envelhecimento são altamente motivados a parar de fumar. (BREITLING et al., 2009, CARAM et al., 2009). Doenças relacionadas ao tabaco, o lazer e o nível de escolaridade foram significativamente associados com os resultados do tratamento, sendo que os indivíduos com um alto risco de fracasso no tratamento eram aqueles sem doença relacionada com o tabaco (CARTERPOKRAS et al., 2011).

A maioria dos pacientes tabagistas pode apresentar alguma comorbidade relacionadas ao tabaco e apresentar sintomas respiratórios. Grande parte da população refere já ter tentado parar de fumar; porém, poucos utilizaram algum recurso para a cessação. (BARRETO et al., 2012).

A prevalência de tabagismo é maior para pessoas com idade $\geq 18$ anos que não têm diploma de ensino médio. Avaliar e relatar a prevalência de tabagismo entre os jovens com idade $<18$ anos que abandonam a escola é fundamental, pois este é o período em que ocorrem problemas de desempenho acadêmico (GARRETT et al., 2013).

As taxas de procura ao tratamento de cessação são consideravelmente maiores em mulheres do que em homens, mesmo que a prevalência do tabagismo é maior entre os homens. É preciso, portanto, o desenvolvimento de estratégias para aumentar 
a consciência de que eles devem procurar ajuda. (AZEVEDO et al, 2011),

Como principais motivos que levariam a cessação: a vontade de realmente sair do vicio; o surgimento de sinais e sintomas provocados ou desencadeados pelo cigarro; preocupações financeiras, gravidez / começar uma família; e desaprovação social. Já no que se refere às razões mais comuns para continuarem com o vício mencionadas: o prazer em fumar, o tédio, a força do hábito, dependência, estresse, ver outras pessoas fumando e a associação com álcool. (UPPAL et al, 2013).

A influencia social serve como forte motivação, bem como fonte de tentação para continuar fumando. Muitos dos entrevistados referiram a influência de companheiros ou colegas, como mais frequente, seguida da influência de familiares. Vício e comportamento habitual e situações de nervosismo, ansiedade, após consumir café, após as refeições, ligações telefônicas longas, amigos que fumam, dirigir por longas distâncias, membros da família que fumam em casa, foram citados como fatores relacionados a continuação do habito de fumar. Evidenciou-se, a partir dos relatos encontrados nos estudos a participação de familiares na iniciação do uso. (CARTERPOKRAS et al., 2011, MARTINS et al., 2011, A. ESTABROOKS et al., 2010).

Problemas de saúde pessoais, o impacto que o cigarro estaria acarretando em crianças e na saúde da família e pressão por parte de outros, foram freqüentemente mencionados motivo para cessação. (CARTER-POKRAS et al., 2011)

O estresse facilita o início do hábito de fumar, diminui a motivação para parar de fumar, e aumentar o risco de recaída. Além disso, as pessoas com depressão ou um transtorno de ansiedade são mais propensos a fumar do que pessoas sem esses transtornos. (W. BRUIJNZEEL, 2012, B. ANSELL et al., 2012, J. R. HUGHES et al., 2009).

N. Doran et al., (2009), ressalta ainda que os indivíduos que não têm planos estabelecidos para parar de fumar podem ser mais propensos a fumar ou a fumar com mais frequiência do que os outros, pelo fato de serem menos propensos a compreender ou acreditar nos efeitos negativos do fumo.

Num estudo realizado por H. Vries et al., (2013), no qual ele avalia os determinantes e os efeitos do planejamento de ações e plano de promulgação de cessação do tabagismo, observou-se que os planos de preparação mais eficazes para a cessação para foram à remoção de todos os produtos do tabaco da casa e escolher uma data certa para parar definitivamente.

Embora grande proporção de fumantes tenha realizado tentativas anteriores de cessação, na maioria das vezes, estudos mostram que eles não participaram de sessões 
estruturadas de aconselhamento e não utilizam nenhum tipo de medicação para o tratamento da dependência, o que está em desacordo com as recomendações das diretrizes para o tratamento do tabagismo. (CARAM et al., 2009).

No que se refere ao estado em que o individuo se encontra em seu comportamento, segundo as Diretrizes para Cessação do tabagismo (2008), os estágios de mudança quando os pacientes iniciam um tratamento para deixar de fumar são divididos em: Précontemplação: Onde não há intenção de parar, nem mesmo uma crítica a respeito do conflito envolvendo o comportamento de fumar. Contemplação: Há conscientização de que fumar é um problema, no entanto, há uma ambivalência quanto à perspectiva de mudança. Preparação: Onde o individuo prepara-se para parar de fumar (quando o paciente aceita escolher uma estratégia para realizar a mudança de comportamento). Ação: Parar de fumar: o paciente toma a atitude que o leva a concretizar a mudança de comportamento. Manutenção: $\mathrm{O}$ paciente deve aprender estratégias para prevenir a recaída e consolidar os ganhos obtidos durante a fase de ação. Neste estágio pode ocorrer a finalização do processo de mudança ou a recaída.

No que diz respeito ao tratamento utilizado para cessação do fumo, para Zhu et al., 2012, vários foram os métodos desenvolvidos para a cessação do tabagismo ao longo do tempo. O uso da farmacoterapia é amplamente utilizado por fumantes que recebem o apoio de profissionais da área da saúde.

Segundo Benowitz (2008), atualmente existem três classes de medicamentos aprovados para a cessação do tabagismo: produtos de reposição de nicotina (adesivo, goma, spray, inalador e losango), bupropiona, e mais recentemente, a vareniclina.

Novos produtos de nicotina que surgiram no mercado nos últimos anos incluem os chamados snus e cigarros eletrônicos (e-cigarros). Estes produtos estão sendo cada vez mais promovidos como alternativas mais seguras para os cigarros tradicionais. Ambos os produtos proporcionam o consumo da nicotina. Snus é uma pequena bolsa de tabaco que libera vapor, que é colocado sob o lábio superior. Ecigarros são dispositivos que funcionam a bateria que se assemelham tanto em tamanho quanto a forma a um cigarro e produzir um vapor que libera nicotina. A critica a esses produtos encontra-se no fato de que mesmo que as pessoas que usam esses produtos não possam ser expostos a todos os produtos químicos nocivos e monóxido de carbono associadas com o tabagismo, não está claro se esses produtos são realmente seguros, pois promovem o consumo continuo, em vez de cessação, além de agir de forma a motivar os jovens que de outra tradicional, não teriam 
começado o uso do tabaco. (HARRILL et al, 2013).

Podemos aliar isso ao conjunto cada vez maior de informações através da melhoria no acesso à internet, telefones celulares e outros dispositivos eletrônicos móveis que abrem novas possibilidades para os fumantes que procuram assistência para cessação. Ocorreu também um aumento de campanhas que favorecem o aumento do preço do cigarro, onde se tenta gerar de certa forma, a diminuição ou a completa cessação do uso, além de mensagens contidas nas próprias embalagens dos cigarros alertando sobre o perigo do cigarro a saúde. (ZHU et al., 2012)

O aumento dos preços dos cigarros é outro fator usado na tentativa de reduzir significativamente tanto o início do uso do tabaco, quanto o consumo por parte dos fumantes. Ainda afirma que as empresas de tabaco têm desafiado a eficácia do aumento de impostos, argumentando que o aumento de impostos vai levar ao aumento do contrabando, o que resultará em menos receita para os governos. Além disso, muitos fumantes acreditam que os aumentos de preços dos produtos do tabaco precisava ser mais drástica a fim de influenciar a motivação e reduzir o número de compras. (SIRICHOTIRATANA et al., 2008, UPPAL et al., 2013)

Atualmente, sabe-se que qualquer que seja o tempo despendido na abordagem à cessação do tabagismo, há um efeito benéfico no processo de interrupção do uso do tabaco. A identificação de outros grupos particularmente suscetíveis à cessação do uso de tabaco, além de os pacientes de maior idade e aqueles com doença crônica, poderia auxiliar na escolha de métodos de abordagem específicos. (SILVA et al., 2011).

Mensagens antitabagismo nos meios de comunicação de massa são um meio para alcançar esse objetivo de cessação do fumo. As intenções dos fumantes para deixar de fumar são um indicador da eficácia dessas mensagens. Porém as campanhas de mídia de massa podem ser ineficazes se não encontrar um limite para a exposição da população suficiente. O conteúdo das mensagens também é importante; mensagens que transmitem os efeitos adversos à saúde do uso do tabaco e exposição à fumaça de segunda mão foram citadas como as mais eficazes do que outros tipos de mensagens. (CAIXETA et al., 2013).

Grande parte dos fumantes tem planos para fazer alterações em seu comportamento de fumante no futuro. Num estudo realizado por Cunningham e Selby (2010), quando perguntado quais eram os seus planos sobre seu comportamento atual, $55 \%$ dos fumantes disseram que planejam parar de fumar, $18,8 \%$ disseram que planejam reduzir e $22,5 \%$ disseram que pretendiam manter, $3,4 \%$ não souberam ou se recusaram; e apenas dois 
entrevistados disseram que iriam aumentar o consumo.

Benowitz (2008), afirma que cerca de $80 \%$ dos fumantes que tentam parar de fumar por conta própria têm alguma recaída no primeiro mês de abstinência, e apenas cerca de $3 \%$ permanecem abstinentes em seis meses. Isto ilustra a força poderosa do tabagismo e da natureza crônica da doença.

São várias as evidencias de que a nicotina seja o principal componente do cigarro responsável pela manutenção da dependência. Sendo que estudos demonstraram que cigarros a partir do qual a nicotina tem sido removida ou aqueles com baixos níveis de nicotina nunca foram amplamente aceitos por parte dos fumantes, enquanto tabaco de mascar e rapé, que apresentam grandes quantidades de nicotina, são amplamente utilizados. (BENOWITZ, 2008)

No entanto segundo Souza et al., (2010), é possível que a dependência de nicotina envolva fatores além da dependência física de nicotina, o que significaria que a dependência de ao cigarro é um conjunto de amplos fatores que abrange várias facetas psicossociais. É possível que a identificação precisa dos fatores distintivos que levam as pessoas a fumar contribua para 0 desenvolvimento de políticas públicas de prevenção e controle do fumo, bem como para o desenvolvimento de estratégias personalizadas para a cessação do tabagismo.

Segundo Caram et al., (2009), os principais fatores associados com o tabagismo e a baixa taxa de tratamento especializado observados estão relacionados com as condições socioeconômicas e educacionais, assim como com a intensidade da dependência entre os indivíduos avaliados.

É provável que grande parte da elevada incidência de cessação do tabagismo encontrada seja decorrente das políticas públicas voltadas para o controle do tabagismo ocorridas no Brasil. Locais onde as ações de saúde para controle do tabagismo são mais intensas contribuem para que ocorra uma menor incidência no numero de tabagistas. (LOPES et al, 2013).

Para Russo et al., (2010), o papel do profissional de saúde torna-se mais relevante à medida que o paciente manifeste o desejo de parar de fumar: em seu estudo constatou-se que $32,1 \%$ dos entrevistados procuraram tratamento após o incentivo médico, contrastando com 9,4\% após o incentivo de familiares. Além disso, 69,8\% dos entrevistados chegaram ao serviço por encaminhamento de um profissional de saúde (os demais chegaram ao serviço por procura espontânea ou por orientação de amigos ou conhecidos). Grande parte dos entrevistados começaram a fumar antes dos 18 anos de idade. Dessa forma, campanhas de prevenção dirigidas e desenhadas especificamente para 
essa faixa etária são necessárias para que possamos ter, no futuro, um panorama mais favorável em relação às taxas de tabagismo na população adulta.

A promoção da cessação do tabagismo deve ser uma prioridade para os profissionais de saúde e para os governos. Ainda há muito a ser realizado. A combinação de intervenções breves, empáticas e apropriadas que combinem o apoio e terapias farmacológicas podem ajudar a incentivar os fumantes a parar e viverem uma vida mais saudável sem fumo. (T. GOODFELLOW et al, 2009, MCIVOR et al., 2009)

A redução da prevalência de fumantes para níveis inferiores aos que existem atualmente garantirá, de forma efetiva, a prevenção de grande parte das doenças crônicas que mais acometem a população brasileira. (SILVA et al, 2011).

$\mathrm{Na}$ literatura médica atual, é imenso o número de artigos, de todas as especialidades, apontando o tabagismo como fator principal de muitas patologias; contudo, pouco se publica sobre o tabagismo em si, seus mecanismos e tratamentos possíveis. (RAMADAM, 2008).

\section{CONSIDERAÇÕES FINAIS}

O cigarro ainda é uma das drogas mais consumidas atualmente, sendo necessárias intervenções não apenas farmacológicas o combate ao habito que é tão prejudicial, mas métodos que envolvam também os diversos aspectos que envolvem o ato de fumar e assim contribuir para um melhor enfrentamento para o combate a esse comportamento de risco.

Existem oportunidades no mundo, para os profissionais de saúde para fornecer apoio e tratamento a fumantes para sair do tabagismo. Para isso todos os pacientes devem ser examinados quanto ao uso do tabaco, para assim melhor prescrever o tratamento ideal para cada caso (HEALTHCARE, 2013).

A revisão de literatura possibilitou identificar o quão difícil é trabalhar com a temática, visto que mesmo que sejam inúmeros os estudos relacionados ao tabagismo, poucos trabalham as percepções dos indivíduos em seu enfrentamento ao vicio.

Conflitos de interesse: Não existem conflitos de interesse entre os autores.

\section{REFERENCIAS}

ANSELL, E. B. et al. Effects of cumulative stress and impulsivity on smoking status.

Hum Psychopharmacol, v. 27, n. 2, p. 2008, Mar 2012. ISSN 0885-6222.

AZEVEDO, Renata Cruz Soares de and FERNANDES, Rejane Firmino.Factors relating to failure to quit smoking: a prospective cohort study. Sao Paulo Med. J. [online]. 2011, vol.129, n.6, pp. $380-386$. ISSN 1516- 
3180. http://dx.doi.org/10.1590/S1516$\underline{31802011000600003 .}$.

BARRETO, Rafael Balsini et al.Tabagismo entre pacientes internados em um hospital universitário no sul do Brasil: prevalência, grau de dependência e estágio motivacional. J. bras. pneumol. [online]. 2012, vol.38, n.1, pp. 72-80. ISSN 18063713. http://dx.doi.org/10.1590/S1806$\underline{37132012000100011 .}$.

BENOWITZ, N. L. Neurobiology of nicotine addiction: implications for smoking cessation treatment. Am J Med, v. 121, n. 4 Suppl 1, p. S3-10, Apr 2008. ISSN 0002-9343.

BREITLING, L. P. et al. Older smokers' motivation and attempts to quit smoking: epidemiological insight into the question of lifestyle versus addiction. Dtsch Arztebl Int, v. 106 , n. 27 , p. 451-5, Jul 2009. ISSN 18660452.

BRUIJNZEEL, A. W. Tobacco addiction and the dysregulation of brain stress systems.

Neurosci Biobehav Rev, v. 36, n. 5, p. 141841, May 2012. ISSN 0149-7634.

CARAM, Laura Miranda de Oliveira et al.Perfil de fumantes atendidos em serviço público para tratamento do tabagismo. $\boldsymbol{J}$. bras. pneumol. [online]. 2009, vol.35, n.10, pp. 980-985. ISSN 1806-

3713. http://dx.doi.org/10.1590/S180637132009001000006.

CARVALHO, Anderson Albuquerque de; GOMES, Lucy and LOUREIRO, Altair Macedo Lahud.Tabagismo em idosos internados em instituições de longa permanência.J. bras. pneumol. [online]. 2010, vol.36, n.3, pp. 339-346. ISSN 18063713. http://dx.doi.org/10.1590/S180637132010000300012.
CARTER-POKRAS, O. D. et al. Barriers and facilitators to smoking cessation among Latino adults. J Natl Med Assoc, v. 103, n. 5, p. 423-31, May 2011. ISSN 0027-968400279684.

CAIXETA et al. Antismoking messages and intention to quit - 17 countries, 2008-2011. MMWR Morb Mortal Wkly Rep, v. 62, n. 21, p. 417-22, May 31 2013. ISSN 01492195.

CUNNINGHAM, J. A.; SELBY, P. L. Future intentions regarding quitting and reducing cigarette use in a representative sample of Canadian daily smokers: implications for public health initiatives. Int J Environ Res Public Health, v. 7, n. 7, p. 2896-902, Jul 2010. ISSN 1660-4601.

DE VRIES, H.; EGGERS, S. M.; BOLMAN, C. The role of action planning and plan enactment for smoking cessation. BMC Public Health, v. 13, p. 393, 2013. ISSN 1471-2458.

Diretrizes para cessação do tabagismo 2008. J. bras. pneumol.[online]. 2008, vol.34, n.10, pp. 845-880. ISSN 18063713. http://dx.doi.org/10.1590/S1806$\underline{37132008001000014}$.

DORAN, N. et al. Impulsivity and cigarette craving: differences across subtypes.

Psychopharmacology (Berl), v. 207, n. 3, p. 365-73, Dec 2009. ISSN 0033-3158. ESTABROOKS, P. A. et al. Defining and understanding success at smoking reduction: a mixed-methods study. Addict Behav, v. 35, n. 12, p. 1113-9, Dec 2010. ISSN 0306-4603.

GARRETT, B. E. et al. Cigarette smoking United States, 2006-2008 and 2009-2010. MMWR Surveill Summ, v. 62 Suppl 3, p. 81-4, Nov 22 2013. ISSN 0892-3787. 
GOULART, D.; ENGROFF, P.; ELY, L. S.; SGNAOLIN, V.; SANTOS, E. F.; TERRA, N. L.; CARLI, G. A. Tabagismo em idosos. Revista Brasileira de Geriatria e Gerontologia, v.13, n.2, pág. 313-320. Rio de Janeiro, 2010.

GOODFELLOW, L. T.; WAUGH, J. B. Tobacco treatment and prevention: what GARRETT, B. E. et al. Cigarette smoking United States, 2006-2008 and 2009-2010. MMWR Surveill Summ, v. 62 Suppl 3, p. 81-4, Nov 22 2013. ISSN 0892-3787.

HARRILL-SMITH, C.; RIPLEY-MOFFITT, C.; GOLDSTEIN, A. O. Tobacco cessation in 2013: what every clinician should know. N C Med J, v. 74, n. 5, p. 401-5, Sep-Oct 2013. ISSN 0029-25590029-2559.

Health-care provider screening for tobacco smoking and advice to quit - 17 countries, 2008-2011. MMWR Morb Mortal Wkly Rep, v. 62, n. 46, p. 920-7, Nov 222013. ISSN 0149-2195.

HUGHES, J. R.; MARCY, T. W.; NAUD, S. Interest in treatments to stop smoking. $\mathbf{J}$ Subst Abuse Treat, v. 36, n. 1, p. 18-24, Jan 2009. ISSN 0740-5472.

LOPES, Claudia $\mathrm{S}$ et al.Influencia de fatores psicossociais na cessacao do tabagismo: evidencias longitudinais no Estudo ProSaude. Rev. Saúde Pública [online]. 2013, vol.47, n.4, pp. 732-739. ISSN 00348910. http://dx.doi.org/10.1590/S00348910.2013047004524.

MARTINS, Karla Cristina; MARIA, Eliane. Mudança do comportamento de fumar em participantes de grupos de tabagismo. Psic.: Teor. e Pesq. vol.27 no.1 Brasília. Jan./Mar. 2011. http://dx.doi.org/10.1590/S010237722011000100008.
MCIVOR, A. et al. Best practices for smoking cessation interventions in primary care. Can Respir J, v. 16, n. 4, p. 129-34, Jul-Aug 2009. ISSN 1198-2241.

\section{OLIVEIRA, J. D. F. Avaliação da} Qualidade de Vida de um Grupo de Tabagistas Participantes de um Programa Interdisciplinar em Centro de Saúde: A Questão da Atividade Física. Dissertação (Mestrado), Mestrado em Educação Física. Pós-Graduação da Faculdade de Educação Física da Universidade Estadual de Campinas, São Paulo, 2008.

PUBMED, PubMed Ajuda. Disponível em: http://www.ncbi.nlm.nih.gov/books/NBK382 7/\#pubmedhelp.PubMed_Quick_Start . Acesso em 06 de dezembro de 2013.

RAMADAM, Zacaria Borge Ali.Tabagismo: dos fundamentos ao tratamento. Rev. psiquiatr. clín. [online]. 2008, vol.35, n.1, pp. 35-36. ISSN 0101-

6083. http://dx.doi.org/10.1590/S010160832008000100008.

REIS, Regiane Costa Martins e; FORTES, Renata Costa. Fatores associados a não cessação do Tabagismo em participantes do Grupo de Terapia de um Centro de Saúde do Distrito Federal. Revisa. 2012; 1(1): 3-8 Jan/Jun 2012. ISSN: 2179-0981.

RONDINA, Regina de Cássia; GORAYEB, Ricardo and BOTELHO, Clóvis.Características psicológicas associadas ao comportamento de fumar tabaco. J. bras. pneumol.[online]. 2007, vol.33, n.5, pp. 592601. ISSN 1806-

3713. http://dx.doi.org/10.1590/S1806$\underline{37132007000500016 .}$.

RUSSO, Adriana Carneiro e AZEVEDO, Renata Cruz Soares de. Fatores motivacionais que contribuem para a busca de tratamento ambulatorial para a cessação do tabagismo em 
um hospital geral universitário. J. bras.

pneumol. [online]. 2010, vol.36, n.5, pp. 603611. ISSN 1806-

3713. http://dx.doi.org/10.1590/S1806$\underline{37132010000500012 .}$

SANTOS, Sergio Ricardo; GONCALVES, Maria Stela; LEITAO FILHO, Fernando Sergio Studart and JARDIM, José Roberto.Perfil dos fumantes que procuram um centro de cessação de tabagismo.J. bras. pneumol. [online]. 2008, vol.34, n.9, pp. 695701. ISSN 1806-

3713. http://dx.doi.org/10.1590/S1806$\underline{37132008000900010 .}$

SILVA, Gulnar Azevedo e; VALENTE, Joaquim Gonçalves e MALTA, Deborah Carvalho.Tendências do tabagismo na população adulta das capitais Brasileiras: uma análise dos dados de inquéritos telefônicos de 2006 a 2009. Rev. bras. epidemiol. [online]. 2011, vol.14, suppl.1, pp. 103-114. ISSN 1415-790X. http://dx.doi.org/10.1590/S1415790X2011000500011.

SCIELO. Disponível em:

http://www.scielo.br/?lng=pt . Acesso em 06 de dezembro de 2013.

SILVA, Rodney Luiz Frare e et al.Cessação de tabagismo em pacientes de um hospital universitário em Curitiba. J. bras.

pneumol. [online]. 2011, vol.37, n.4, pp. 480487. ISSN 1806-

3713. http://dx.doi.org/10.1590/S180637132011000400010.
SIRICHOTIRATANA, N. et al. Prevalence of smoking and other smoking-related behaviors reported by the Global Youth Tobacco Survey (GYTS) in Thailand. BMC Public Health, v. 8 Suppl 1, p. S3, 2008. ISSN 1471-2458.

SOUZA, E. S. et al. University of Sao Paulo Reasons for Smoking Scale: a new tool for the evaluation of smoking motivation. J Bras Pneumol, v. 36, n. 6, p. 768-78, Nov-Dec 2010. ISSN 1806-3713.

UPPAL, N. et al. The forgotten smoker: a qualitative study of attitudes towards smoking, quitting, and tobacco control policies among continuing smokers. BMC Public Health, v. 13, p. 432, 2013. ISSN 1471-2458

ZHU, S.; LEE, M.; ZHUANG, Y.; GAMST, A.; WOLFSON, T. Interventions to Increase Smoking Cessation at the Population Level: How Much Progress Has Been Made in the Last Two Decades? Tobacco Control. Março, 2012; v.21, n. 2, pag. 110-118. 2012. 